\title{
Selective Parent Ion Axialization for Improved Efficiency of Collision-Induced Dissociation in Laser Desorption-Ionization Fourier Transform Ion Cyclotron Resonance Mass Spectrometry
}

\author{
Troy D. Wood, ${ }^{*}$ Charles W. Ross, $\mathrm{II}_{1}^{\dagger}$ and Alan G. Marshall, ${ }^{\dagger}$ \\ Department of Chemistry, The Ohio State University, Columbus, Ohio, USA
}

\begin{abstract}
We have systematically established the excitation frequency, amplitude, duration, and buffer gas pressure for optimal axialization efficiency and mass selectivity of quadrupolar excitation-collisional cooling for isolation of parent ions for collision-induced dissociation in Fourier transform ion cyclotron resonance mass spectrometry. For example, at high quadrupolar excitation amplitude, ion axialization efficiency and selectivity are optimal when the applied quadrupolar excitation frequency is lower than the unperturbed ion cyclotron frequency by up to several hundred hertz. Moreover, at high buffer gas pressure $\left(10^{-6}\right.$ Torr), quadrupolar excitation duration can be quite short because of efficient collisional cooling of the cyclotron motion produced by magnetron-to-cyclotron conversion. Efficiency. detected signal magnitude, and mass resolving power for collision-induced dissociation (CID) product ions are significantly enhanced by prior parent ion axialization. With this method, we use argon CID to show that $\mathrm{C}_{94}^{+}(m / z$ 1128) formed by Nd:YAG laser desorption-ionization behaves as a closed-cage structure. (J Am Soc Mass Spectrom 1994, 5, 900-907)
\end{abstract}

$\mathrm{I}$ Fourier transform ion cyclotron resonance (FTICR) mass spectrometry it is well known that collisions between high mass ions and low mass neutrals can effectively cool the high amplitude cyclotron motion (i.e., reduce the cyclotron radius) of high mass ions without complete loss of ions from the trap [1, 2]. Of the three ion motional modes (cyclotron rotation, magnetron rotation, and trapping oscillation [3]), the cyclotron and axial motional amplitudes are reduced by ion-neutral collisions because of the stable nature of their motions (i.e., as ions lose cyclotron or axial energy, they relax to the bottom of their respective potential wells). Magnetron motion, however, is inherently unstable (i.e., the potential energy is a maximum, not a minimum, at the center of the trap); thus, as an ion loses energy by ion-neutral collisions, its magnetron radius increases. Two undesirable effects of collisioninduced magnetron orbit expansion result: First, ions cannot be transferred to the analyzer in a dual-trap instrument [4] (for detection at lower pressure for

Address reprint requests to Dr. Alan G. Marshall, National High Magnetic Field Laboratory, Florida State University, 1800 East Paul Dirac Drive, Tallahassee, FL 32306-4005.

* Present Address: Baker Laboratory, Dept. of Chemistry, Comell University, Ithaca, NY 14853-1301.

${ }^{\dagger}$ Present Address: National High Magnetic Field Laboratory, Florida State University, 1800 East Paul Dirac Drive, Tallahassee, FL 32306-4005 higher mass resolving power [5]) once collisions have increased the ion magnetron radius to a value larger than the radius of the conductance limit between the two traps; second, ions are eventually lost by radial diffusion [6] when the ion magnetron radius reaches the radius of the trap.

Thus, an obvious (in retrospect!) solution to the magnetron radial diffusion problem is to convert magnetron motion into cyclotron motion. In the presence of ion-neutral collisions, the cyclotron motion then will be collisionally damped and ions will relax to the $z$-axis (magnetic field direction) of the ion cyclatron resonance (ICR) ion trap. In a purely quadrupolar trapping potential, the desired magnetron-to-cyclotron conversion may be accomplished by application of an azimuthal quadrupolar excitation potential at the combination frequency $\omega_{\mathrm{c}}=\omega_{+}+\omega_{-}$, in which $\omega_{+}$is the reduced ion cyclotron frequency, $\omega_{-}$is the ion magnetron frequency, and $\omega_{\varepsilon}=q B / m$ ( $B$ is magnetic field induction in tesla and $m / q$ is the ion mass-to-charge ratio in SI units) is the unperturbed ion cyclotron frequency in the absence of an electrostatic field. First demonstrated for axialization of ions of a single massto-charge ratio in a Penning trap $[7,8]$, the axialization method subsequently has been adapted for FTICR mass spectrometry of ions that have a range of mass-tocharge ratios [9].

The quadrupolar excitation-collisional cooling axi- 
alization technique offers several important advantages for FTICR mass spectrometry:

1. Higher (factor of up to 100 ) signal-to-noise ratio and lower detection limit with improved peak shape $[9$, $10]$.

2. Improved mass resolving power (up to a factor of 500) [10].

3. Higher mass selectivity in the first stage of tandem mass spectrometry [10].

4. Higher product ion mass resolving power in the second stage of tandem mass spectrometry [11].

5. Improved transfer efficiency to move ions through a narrow conductance limit (i.e., more efficient differential pumping with fewer stages).

6. Improved ion remeasurement efficiency (up to 99\%) for an additional increase in signal-to-noise ratio and/or multiple experiments with a single trappedion packet [12] remeasurement of heavy ions has also been achieved without axialization, but at lower efficiency (in a cubic trap [1]) or in an axially elongated trap (with lower radially outward-directed electric field) [13].

7. Extended trapping period to allow for thousands of ion-neutral collisions to release excess ion internal electronic or vibrational excitation energy for efficient cooling of ions [9].

By analogy to a two-level quantum mechanical system, Guan and co-workers [14, 15] predicted and demonstrated that broadband axialization may be achieved by application of repeated low amplitude frequencysweep or stored-waveform inverse Fourier transform (SWIFT) quadrupolar excitation in the presence of ionneutral collisions.

As described in several recent papers $[10,12,16$, 17], quadrupolar excitation-collisional cooling for ion axialization has been applied to high mass singly charged ions formed by laser desorption. These reports showed that laser-desorbed ions may be trapped for a long period of time and measured with high mass resolving power. The increased mass resolving power results not only from detection in a low pressure trap, but also from thermalization of the laser-desorbed ions, which narrows their $z$-axis distribution so that all ions experience near-identical preexcitation and postexcitation electrostatic and magnetic fields [10]. The effect of space charge has been noted $[10,16]$ and a digital filtering technique has been applied to improve mass resolving power (which can be degraded under high space charge conditions) [16]. Perhaps most important for analytical applications is that high ion isolation selectivity [7-10] that surpasses that of SWIFT radial ejection [18-21] (for high mass ions) has been observed for the quadrupolar excitation-collisional axialization technique (e.g., isolation of just the ${ }^{12} \mathrm{C}$ isotope for the ionic species $\mathrm{C}_{84}$ from a mixture of fullerenes [10]). The potential of the observed high ion selectivity in tandem mass spectrometry experiments was immediately noted [10].

In this paper, we systematically examine the effect of the relevant experimental parameters (i.c., applied quadrupolar excitation frequency, duration, and amplitude; buffer gas pressure) on the efficiency and mass selectivity of ion axialization. We demonstrate the results on high mass singly charged ions produced by laser desorption-ionization FTICR mass spectrometry. Moreover, we show that axialized parent ions may be transferred efficiently to the analyzer trap for high resolution collision-induced dissociation (tandem mass spectrometry [22]) analysis.

\section{Experimental}

\section{Sample preparation}

The fullerene sample used to examine the effects of the various axialization-cooling parameters was generated from $\mathrm{KH}$ soot [23] and subsequently was transferred to a pressure bomb with toluene and heated to $275^{\circ} \mathrm{C}$ to form giant fullerenes [24]. The fullerene sample used in the tandem mass spectrometry experiment was prepared by sublimation of fullerene soot originally generated from graphite electrodes by the arc-welding technique (described by Smalley and co-workers [25]) onto a pellet of $\mathrm{KBr}$.

\section{FTICR Mass Spectrometry Parameters}

All experiments were carried out on an Extrel Millipore FTMS-2000 instrument (Extrel FTMS, Madison, WI) equipped with Helix Technology CryoTorr-8 2000 L/s cryopumps (Waltham, MA) for both source and analyzer vacuum chambers, operated at a magnetic field of $3.02 \mathrm{~T}$ and equipped with two $4.76-\mathrm{cm}$ cubic traps in a standard dual-trap configuration. Collision buffer gas for ion cooling (argon) was admitted into the source compartment through a leak valve (Model 951-5100, Varian, Palo Alto, CA) connected to the batch inlet system of the instrument. Collision-induced dissociation (CID) collision gas (argon) was admitted into the analyzer compartment by means of a pulsed solenoidal valve. A YG-660A Nd:YAG laser (Continuum, Santa Clara, CA) was used to produce singleshot fundamental $(1.064-\mu \mathrm{m}, 120-\mathrm{mJ} / \mathrm{pulse})$ and $\mathrm{sec}-$ ond harmonic (532-nm, 80-mJ/pulse) pulses of $\sim 10-\mathrm{ns}$ duration over an area of $\sim 0.2 \mathrm{~mm}^{2}$ when directed at the sample at an incident angle of $\sim 30^{\circ}$. The mechanical and electronic interfaces between the mass spectrometer and the laser have been described elsewhere [26].

The event sequence and the corresponding wiring diagram for the axialization process (azimuthal quadrupolar excitation in the presence of a buffer gas for collisional cooling) in a dual-trap arrangement were described in a previous paper [9]. The experimental event sequence for tandem mass spectrometry experi- 
ments conducted after selective ion axialization includes an additional pulsed valve event followed by a parent ion dipolar excitation event sandwiched between the ion source-to-analyzer transfer and broadband dipolar excitation-dipolar detection events. Initially, we applied azimuthal quadrupolar excitation at the unshifted (by trapping potential) cyclotron frequency $\omega_{c}=q B / m$, of the ion(s) of interest for several seconds to axialize the irradiated ions by converting magnetron motion into cyclotron motion in the presence of simultaneous cooling of the cyclotron motion by ion-neutral collisions [8]. However, we soon confirmed that the maximum detected signal does not result necessarily from quadrupolar irradiation at $\omega_{c}$ [9] and that optimal mass selectivity is achieved at applied axialization frequency several hundred hertz lower than $\omega_{c}$ (see Results and Discussion).

In the present experiments, axialization was initiated promptly after laser desorption (LD)-ionization. After axialization, the conductance limit voltage was lowered to allow for ion transfer from the source trap to the analyzer trap (Figures 1-4, $210 \mu \mathrm{s}$; Figure 5, 223 $\mu \mathrm{s})$. After ion transfer, the conductance limit voltage was returned to the original trapping voltage $(+3 \mathrm{~V}$ in all LD-FTICR mass spectra except Figure $5 a,+1$ V). A pulsed valve was used to admit a burst of argon $\left(1-5 \times 10^{-6}\right.$ torr $)$ for collision-induced dissociation (CID) experiments [27-30] in the analyzer trap. Pressures in the source and analyzer traps during cooling and detection were varied and are noted in the figure legends. Frequency-sweep excitation from $5-500 \mathrm{kHz}$ (sweep rate $400 \mathrm{~Hz} / \mu \mathrm{s}$ ) was applied at an amplitude of $61 \mathrm{~V}$ peak to peak $(\mathrm{p}-\mathrm{p})$. Detection was direct mode (200-kHz Nyquist bandwidth) to yield $32 \mathrm{~K}$ time-domain data points, which were padded with an equal number of zeroes and subjected to discrete Fourier transformation without prior apodization.

\section{Results and Discussion}

\section{Effect of Quadrupolar Excitation Amplitude on Axialization Efficiency and Mass Selectivity}

The amplitude of the applied quadrupolar excitation dramatically affects ion isolation selectivity in the LD-FTICR mass spectra of a fullerene mixture. At low quadrupolar excitation amplitude $[1.6 \mathrm{~V}(\mathrm{p}-\mathrm{p})]$ at 45.1 $\mathrm{kHz}$ ( $\nu_{\mathrm{c}}$ for ${ }^{12} \mathrm{C}_{86}^{+}$is $44.94 \mathrm{kHz}$ at $3.0 \mathrm{~T}$ ) for $30 \mathrm{~s}$ at $1.1 \times 10^{-5}$-torr argon pressure in the source half of a dual trap, only $\mathrm{C}_{86}^{+}$could be transferred to the analyzer trap $\left(5.9 \times 10^{-8}\right.$ torr) and detected, but with low signal-to-noise ratio (Figure 1a). At higher quadrupolar excitation amplitude $(11 \mathrm{~V}(\mathrm{p}-\mathrm{p}))$, the predominant fullerene cluster observed is $\mathrm{C}_{84}^{+}\left(\nu_{\mathrm{c}}\right.$ of ${ }^{12} \mathrm{C}_{84}^{+} \sim 46 \mathrm{kHz}$ ), with a little $\mathrm{C}_{82}^{+}$(Figure 1b). A further increase in the quadrupolar excitation amplitude (18 V (p-p)) leads to almost complete isolation of ${ }^{12} \mathrm{C}_{84}^{+}$at high signal-tonoise ratio (Figure $1 \mathrm{c}$ ). Some $\mathrm{C}_{82}^{+}$ions also are transferred to the analyzer trap. Finally, at even higher quadrupolar excitation amplitude (32 V ( $p-p)$ ), ions of several nominal mass-to-charge ratios are axialized and transferred to the analyzer trap (Figure 1d). Of the observed fullerenes, $\mathrm{C}_{78}^{+} \mathrm{C}_{80}^{+} \mathrm{C}_{82}^{+}$and $\mathrm{C}_{84}^{+} \mathrm{C}_{80}^{+}$is the most abundant, even though $v_{c}$ for ${ }^{12} \mathrm{C}_{80}^{+}$is $48.31 \mathrm{kHz}$, which is more than $3 \mathrm{kHz}$ above the applied quadrupolar excitation frequency, $45.1 \mathrm{kHz}$.

These results may be understood from the predicted amplitude dependence of quadrupolar excitation at $\omega_{c}$ in the presence of collisional damping, as first explained in detail for the related case of $x z$ quadrupolar excitation [31] and more recently for azimuthal quadrupolar excitation (Guan, S.; Kim, H. S.; Marshall, A. G.; Wahl, M. C.; Wood, T. D.; Xiang, X., unpublished). Above a critical excitation amplitude

$$
V_{(\mathrm{p}-\mathrm{p})}=m a^{2} \gamma \omega_{c} / 3 q \alpha
$$

in which $\gamma$ is the frictional damping rate constant (per second) and a is 2.77373 for a cubic trap, the magnetron radius and cyclotron radius each damp exponentially at the same rate constant, and there is continuous periodic interconversion between magnetron and cyclotron motions at interconversion frequency $\Omega / 2$ $[32,33]$ :

$$
\frac{\Omega}{2}=\frac{3 \alpha q V_{(\mathrm{p}-\mathrm{p})}}{4 m a^{2} \omega p}
$$

in which $\alpha$ is a geometric constant $(2.77373$ for a cubic trap), $q$ is ionic charge (coulombs), $V_{(p-p)}$ is the quadrupolar excitation voltage amplitude, $m$ is the ionic mass (kilograms), $a$ is the trap edge dimension

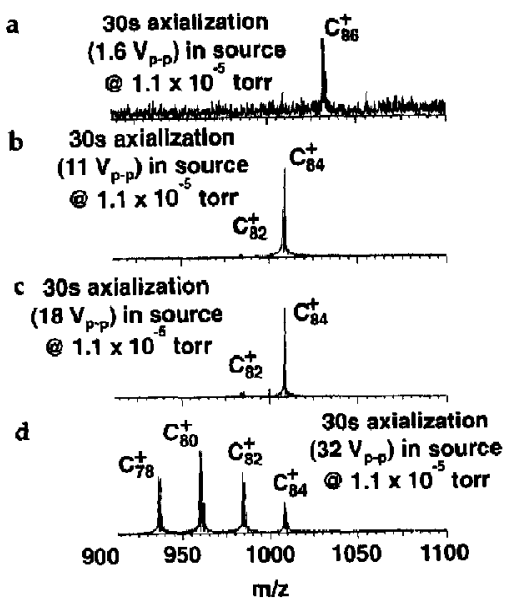

Figure 1. Nd:YAG laser desorption-ionization FTICR mass spectra of fullerene soot ions cooled and axialized in the source half of a dual trap at different applied quadrupolar excitation amplitudes. Proseeding from top to bottom, single-frequency excitation voltage is (a) 1.6 , (b) 11 , (c) 18 , and (d) $32 \mathrm{~V}$ (p-p) at $45.1 \mathrm{kHz}$ for $30 \mathrm{~s}$ at $1.1 \times 10^{-5}$ torr, with subsequent transfer of ions to the analyzer trap for dipolar excitation-dipolar detection at $5.9 \times 10^{-8}$ torr. 
(meters), and $\omega_{\mathrm{p}}$ is the "parametric" frequency $\omega_{+}-$ $\omega_{-}$. Complete conversion of magnetron motion to cyclotron motion occurs when the quadrupolar excitation period $\left(T_{\text {conversion }}\right)$ is given by

$$
T_{\text {conversion }}=\frac{\pi}{\Omega}
$$

Below the critical excitation amplitude of eq 1 there is "critical damping": that is, the magnetron and cyclotron radii vary exponentially (with different rate constants), but there is no interconversion between the two modes. Finally, at very low excitation amplitude the magnetron radius expands slowly and exponentially, while the cyclotron radius damps rapidly and exponentially. Thus, at very low quadrupolar excitation amplitude, ions are not axialized because the magnetron radius continues to expand exponentially. At intermediate excitation amplitude, the magnetron radius decreases exponentially (and at a slower rate than the exponential decrease of the cyclotron radius), but ions are not "heated" significantly because their magnetron (potential) energy never is converted into cyclotron (kinetic) energy. Above the critical excitation amplitude ions do gain kinetic energy by magnetronto-cyclotron conversion, so that ion-neutral collisions are suprathermal in energy. Thus there is an optimal quadrupolar excitation amplitude: too low amplitude fails to axialize the ions and too high amplitude gives initially off-axis ions so much kinetic energy that they may react or collisionally dissociate on collision with neutrals.

We may therefore qualitatively understand the behavior in Figure 1 by considering the excitation amplitude Fourier component magnitude at $\omega_{c}$. For a given collision gas pressure and above-critical excitation voltage amplitude, the optimal excitation amplitude at $\omega_{\mathrm{r}}$ will be produced when the excitation frequency is offset from $\omega_{\mathrm{c}}$. For single-frequency excitation, the offset will be determined by the $\sin (\omega T) / \omega$ frequency-domain profile of the excitation itself [34]. For frequency-sweep or SWIFT excitation, the frequency-domain profile of the excitation is more complex [35-37]. Thus, at sufficiently high quadrupolar excitation amplitude the frequency-domain magnitude spectrum of the excitation spans a wide range of frequency, and ions of a range of $\omega_{c}$ (and thus a range of mass-to-charge ratios) may be axialized (Figure 1d). However, because high amplitude excitation also heats initially off-axis ions, those ions may collisionally dissociate, which explains the appearance of ions of lower (but not higher) mass-to-charge ratio than $\mathrm{C}_{84}^{+}$that result from high amplitude quadrupolar irradiation at the unperturbed cyclotron frequency of $\mathrm{C}_{84}^{+}$(Figure $1 \mathrm{~d})$. The present results are consistent with those previously reported by Schweikhard and coworkers [9], who found the highest trapping efficiency of axialized mass-selected ions at quadrupolar excitation amplitude of $1-12 \mathrm{~V}(\mathrm{p}-\mathrm{p})$.

\section{Effect of Quadrupolar Excitation Frequency on Axialization Efficiency and Mass Selectivity}

As explained above, axialization produced by high amplitude azimuthal quadrupolar excitation in the presence of ion-neutral collisions may be effective even when the quadrupolar irradiation frequency is several kilohertz removed from $\nu_{\mathrm{c}}=\nu_{+}+\nu_{-}$of benzene molecular $\mathrm{C}_{6} \mathrm{H}_{6}^{+}$ions [9]. Here, we report that optimal selective ion isolation for a given ion mass-to-charge ratio is achieved at applied quadrupolar excitation frequency significantly below even the reduced (i.e., observed) ion cyclotron frequency $\nu_{+}=\nu_{c}-\nu_{-}$.

This effect is nicely exhibited in Figure 2, which shows FIICR magnitude-mode spectral peak areas for fullerene ions (from the $\mathrm{KH}$ soot extracted in a pressure bomb) as a function of the applied quadrupolar excitation frequency $(44-46 \mathrm{kHz})$. In these experiments, argon in the source trap was maintained at $5 \times 10^{-7}$ torr and detection was performed in the analyzer at $0.3 \times 10^{-8}$ torr. The total axialization period was $30 \mathrm{~s}$. Optimal mass selectivity for the $\mathrm{C}_{84}^{+}$ion occurred at an applied quadrupolar excitation frequency of $44.7 \mathrm{kHz}$, compared to an unperturbed cyclotron frequency for ${ }^{12} \mathrm{C}_{84}^{+}$of $\sim 46 \mathrm{kHz}$. At this excitation frequency, axialization is highly mass selective: no other fullerene ions are transferred and detected in the analyzer. Interestingly, optimal ion selection does not seem to occur at the same frequency as optimal ion abundance (measured from magnitude-mode peak area), which occurs at $45.3 \mathrm{kHz}$ for $\mathrm{C}_{84}^{+}$. Complete isolation was not achieved for $\mathrm{C}_{86}^{+}$within the range of applied excitation frequency. The unperturbed $\mathrm{cy}^{-}$ clotron frequency of $\mathrm{C}_{86}^{+}$is $44.94 \mathrm{kHz}$, whereas the maximal observed ion signal for $\mathrm{C}_{86}^{+}$was at $44.2 \mathrm{kHz}$.

In addition to the excitation amplitude effect, another explanation for our observed results may be that the quadrupolar excitation-collisional cooling method

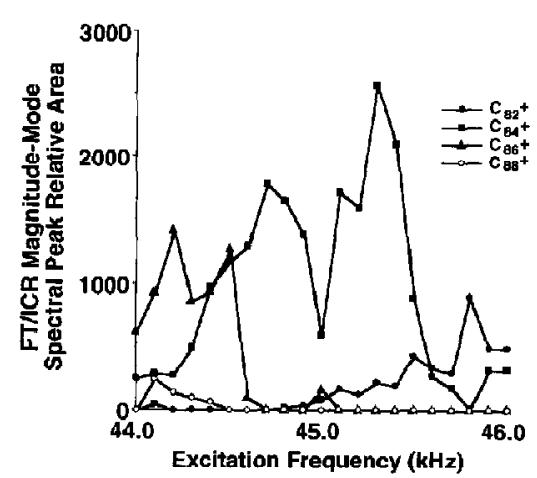

Figure 2. FTICR magnitude-mode spectral peak area for each of several Nd:YAG laser desorbed-ionized fullerene cations as a function of applied quadrupolar excitation frequency in the range from 44 to $46 \mathrm{kHz}$, for fixed quadrupolar excitation amplitude ( 4 $\mathrm{V}(\mathrm{p}-\mathrm{p}))$ and duration (30 s), at fixed source trap pressure of $5 \times 10^{-7}$ torr, followed by ion transfer to the analyzer trap for dipolar excitation-dipolar detection at $0.3 \times 10^{-8}$ torr. 
can be so effective in driving ions to the center of the ICR trap that the high space charge from the resulting highly concentrated ion packet distorts the otherwise near-perfect quadrupolar trapping potential near the center of the trap. Space charge adds to the electrostatic trapping potential and (unless the ion packet happens to have the shape of an ellipsoid of revolution [38]) warps the electrostatic potential to nonquadrupolar form. In support of this view, time-dependent cyclotron frequency shifts and peak distortion have been observed independently for axialized ions, and the effect appears to be larger for more abundant ions $[7,9,10,16]$.

\section{Effect of Buffer Gas Pressure on Axialization Efficiency and Mass Selectivity}

Equation 1 and the related discussion show that the efficacy of axialization is determined by the combined effect of quadrupolar excitation amplitude and collisional damping rate. Thus just as axialization efficiency and mass selectivity at a given collision gas pressure are optimized at a particular quadrupolar excitation frequency and amplitude (at $\omega_{c}$ ), efficiency and selectivity at a given excitation frequency and amplitude will be optimized at a particular collision gas pressure, as seen in Figure 3 for laser desorbed-ionized fullerene cations. In Figure 3, the amplitude $(4 \mathrm{~V}(\mathrm{p}-\mathrm{p}))$, frequency $(45.1 \mathrm{kHz})$, and duration ( $30 \mathrm{~s})$ of the applied quadrupolar excitation are fixed, and the argon pressure in the source trap is varied. Ions are transferred to the analyzer trap and detected at a background pressure of $0.4 \times 10^{-8}$ torr, $\nu_{c}$ is $44.94 \mathrm{kHz}$ for ${ }^{12} \mathrm{C}_{86}^{+}$and $46 \mathrm{kHz}$ for ${ }^{12} \mathrm{C}_{84}^{+}$. At low argon source-trap pressure

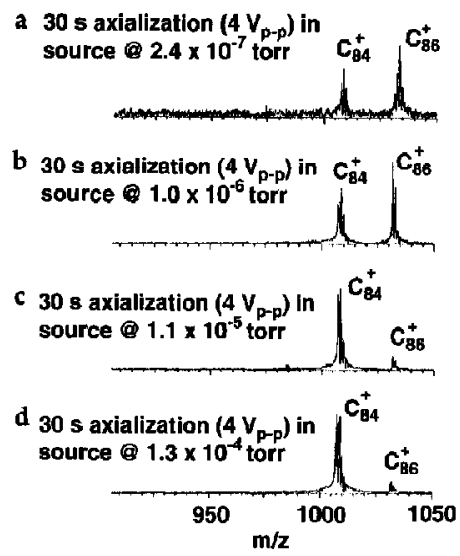

Figure 3. Nd:YAG laser desorption-ionization FTICR mass spectra of fullerene soot ions axialized for $30 \mathrm{~s}$ in the source trap at different pressure, at fixed quadrupolar excitation amplitude $(4 \mathrm{~V}(\mathrm{p}-\mathrm{p}))$ and frequency $(45.1 \mathrm{kHz})$. Proceeding from top to bottom, suurce and analyzer trap pressures are (a) $2.4 \times 10^{-7}$ and $0.2 \times 10^{-8}$ torr, (b) $1.0 \times 10^{-6}$ and $0.7 \times 10^{-8}$ torr, (c) $1.1 \times 10^{5}$ and $5.9 \times 10^{-8}$ torr, and (d) $1.3 \times 10^{-4}$ and $6.1 \times$ $10^{-7}$ torr.
( $2.4 \times 10^{-7}$ torr), fullerene cluster ions $\mathrm{C}_{84}^{+}$and $\mathrm{C}_{86}^{+}$are observed, but at low abundance (Figure 3a), presumably because the collisional damping of the cyclotron motion is too slow to axialize the ions for efficient transfer through the 2-mm conductance limit to the analyzer trap. Increasing the argon source-trap pressure increases the axialization efficiency for both $\mathrm{C}_{84}^{+}$ and $\mathrm{C}_{866}^{+}$but with increased dissociation of $\mathrm{C}_{86}^{+}$due to more collisions during the axialization period. Thus, higher argon pressure at first improves the efficiency of axialization (by increasing the damping rate for cyclotron motion), but at sufficiently high pressure the quadrupolar excitation amplitude is insufficient to overcome the magnetron radius expansion and axialization efficiency is reduced.

A lower mass buffer gas (e.g., helium) could be used to minimize CID during the axialization period (prior to ion transfer to the analyzer trap for subsequent CID with argon) because the center-of-mass collision energy would be too low to activate dissociation of ions of $m / z 1000$. Unfortunately, helium buffer gas is not well suited to our system because helium is not efficiently removed by our cryopumps.

\section{Effect of Quadrupolar Excitation Period on Axialization Efficiency and Mass Selectivity}

At a given buffer gas pressure, the number of ion-neutral collisions is proportional to the duration of the quadrupolar excitation period. Figure 4 shows the dependence of axialized ion relative abundance on excitation period, for fixed quadrupolar excitation frequency $(45.1 \mathrm{kHz})$ and amplitude $(18 \mathrm{~V}(\mathrm{p}-\mathrm{p}))$ and argon source-trap pressure $\left(1.0 \times 10^{-5}\right.$ torr $)$, with analyzer trap detection at $5.9 \times 10^{-8}$ torr. Under these conditions, the predominant observed species is $\mathrm{C}_{84}^{+}$. Ion isolation efficiency and selectivity are relatively independent of the number of ion-neutral collisions above a relatively low threshold: maximal ion signal is achieved after only about $5 \mathrm{~s}$ of quadrupolar excitation. Similar behavior has been reported by

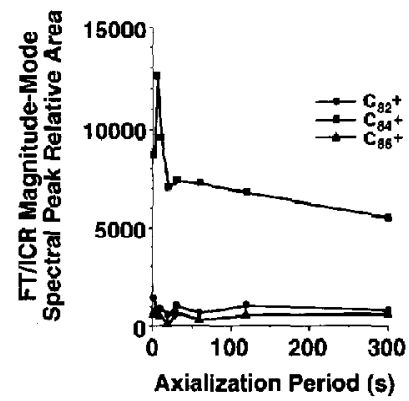

Figure 4. Nd:YAG laser desorption-ionization FTICR magnitude-mode spectral peak area for each of several fullerene cations as a function of quadrupolar irradiation period. In each case, argon pressure in the source trap was $1 \times 10^{-5}$ torr, with quadrupolar excitation $(18 \mathrm{~V}(\mathrm{p}-\mathrm{p}))$ applied at $45.1 \mathrm{kHz}$. 
Schweikhard and coworkers [9] for benzene molecular cations. It appears that once the magnetron radius has been reduced to near zero, no further improvement is found, and ions are in fact slowly lost, perhaps due to axial ejection arising from the increased space charge that results from compaction of the ion cloud at the center of the ion trap.

\section{Tandem Mass Spectrometry: Collision-Induced Dissociation of Axialized Parent Ions}

Tandem mass spectrometry has proved extremely useful for elucidating molecular structure, particularly for high mass ions [22]. In a tandem mass spectrometry experiment, parent ions of a given mass-to-charge ratio are selected and activated to fragment into product ions, whose masses reveal structural features of their parent ion. The FTICR mass spectrometer is extremely well-suited to perform tandem mass spectrometry experiments [39] because FTICR mass spectrometers are multichannel instruments (rapid spectral collection or high signal-to-noise ratio in a short period versus scanning instruments), all FTICR tandem mass spectrometry experiments take place in time rather than space because ions are trapped (thus scattering losses typical of other mass analyzers are minimized; also, confinement of ions in a trap allows FTICR MS ${ }^{n}$ experiments $(n>2)$ to be performed if needed $[30,40,41])$; and mass resolving power and mass accuracy are unsurpassed [11].

The ultimate effectiveness of FTICR tandem mass spectrometry [42] lies in the ion selection process, wherein ions of (typically) a single mass-to-charge ratio may be "filtered" from other ions in the trap by ejecting the unwanted ions. Until recently, the best method for selecting parent ions of a given mass-tocharge ratio was SWIFT radial ejection of ions of all other mass-to-charge ratios [43], because SWIFT excitation offers optimal if irradiation selectivity. Moreover, SWIFT radial excitation also has proved effective in the recently developed two-dimensional stored waveform ion modulation [44] and Hadamard transform tandem mass spectrometry techniques [45].

However, SWIFT radial ejection has demonstrated difficulty in nominal mass isolation of species of $m / z$ $>1000$, presumably because of the shift in ICR frequency with increasing ion cyclotron orbital radius. Thus even if the rf radial excitation is precise to within $\ll 1 \mathrm{~Hz}$, the ions of interest will drift out of resonance during the excitation process. Fortunately, axialization by quadrupolar excitation relies on forcing ions of the desired mass-to-charge ratio to the center of the trap (where the rf field is spatially uniform and the electrostatic trapping potential is nearly perfectly quadrupolar) and allowing ions of all other mass-to-charge ratios to diffuse radially outward by virtue of ion-neutral collisions. Thus, quadrupolar axialization in the presence of ion-neutral collisions is in principle much more mass selective in isolating high mass parent ions of a given mass-to-charge ratio, as shown by prior successful isolation of monoisotopic ${ }^{12} \mathrm{C}_{84}^{+}(m / z=1008)$ from a fullerene mixture [10]. Here we apply the axialization method for isotopically selective tandem mass spectrometry of parent ions of even higher mass-tocharge ratio.

In the present experiments, parent ions are isolated by single-frequency axialization of parent ions of a given mass-to-charge ratio in the source trap, followed by transfer of (only) those parent ions to the analyzer trap. A quick burst of argon $\left(\sim 1-5 \times 10^{-6}\right.$ torr) is admitted into the analyzer trap. The parent ions are then resonantly (single-frequency) excited to larger ICR orbital radius with correspondingly higher translational kinetic energy $[35,46]$

$$
\text { kinetic energy }=\frac{m u_{x y}^{2}}{2}=\frac{q^{2} B_{0}^{2} r^{2}}{2 m} \quad \text { (SI units) }
$$

in which $v_{x y}$ is the radial ion velocity, $r$ is the postexcitation cyclotron orbital radius, and $B_{0}$ is the applied magnetic field strength (teslas). The final postexcitation ion radius may be determined from

$$
r=\frac{\beta E_{0} T_{\text {excite }}}{2 B_{0}}
$$

where $E_{0}$ is the excitation electric field amplitude (volts per meter), $T_{\text {excite }}$ is the excitation duration (seconds), and $\beta$ corrects for the noninfinite parallel plate configuration of the trap $(\beta \approx 0.79$ for the present 4.76-cm cubic trap at $+3-\mathrm{V}$ trapping voltage) $[46-48]$.

For a heavy ion, high translational energy is required to induce dissociation by collision with a light background neutral gas, because a large molecule can accommodate more energy prior to dissociation (due to its large number of vibrational modes) and because energy transfer becomes increasingly inefficient as the difference between the ion mass and neutral mass increases:

$$
E_{\mathrm{cm}}=E_{\mathrm{lab}} \frac{m_{\mathrm{n}}}{m_{\mathrm{n}}+m_{\mathrm{i}}}
$$

in which $m_{n}$ is the mass of the neutral gas, $m_{\mathrm{i}}$ is the mass of the parent ion, $E_{\text {lab }}$ is the parent ion translational energy (we take the neutral as stationary), and the center-of-mass energy $E_{\mathrm{cm}}$ is the amount of energy available for internal excitation of the ion. Equation 6 shows that FTICR can provide only limited translational energy for CID of singly charged ions of $m / z>$ 1000. Of course, multiple collisions between the parent ion and the CID gas make parent ion dissociation feasible at reduced laboratory and center-of-mass frame energies $[27,49]$.

Our first attempted FTICR tandem mass spectrometry based on the axialization method for parent ion selection was the carbon cluster ion $\mathrm{C}_{94}^{+}$, which is presumed to have a fullerene structure in the solid 
state [50, 51]. A broadband laser desorption FTICR mass spectrum of fullerene-rich soot sublimed onto a $\mathrm{KBr}_{\mathrm{r}}$ pellet shows $\mathrm{C}_{94}^{+}$is present in the soot (Figure 5a). After admission of argon into the source trap $(7 \times$ $10^{-7}$ torr), ion axialization and cooling were performed to isolate $\mathrm{C}_{94}^{+}$(Figure 5b). Optimal ion selectivity was achieved at a quadrupolar excitation frequency of $40.400 \mathrm{kHz}\left(\nu_{\mathrm{c}}\right.$ for ${ }^{12} \mathrm{C}_{94}^{+} \mathrm{m} / \mathrm{z} 1128$, at $3.02 \mathrm{~T}$ is $41.122 \mathrm{kHz}$ ). Following transfer to the analyzer trap, CID was performed with a quick burst of argon ( 1-5 $\times 10^{-6}$ torr) followed by dipolar radial excitation of the $\mathrm{C}_{94}^{+}$ion at the reduced cyclotron frequency $\nu_{+}$of ${ }^{13} \mathrm{C}^{12} \mathrm{C}_{93}^{+}$(i.e., to be sure to excite all of the most abundant isotopes of $\mathrm{C}_{94}^{+}$) from 100 to $1000 \mu$ S (i.e., 3.5 $\mathrm{eV}<$ laboratory frame energy $<353 \mathrm{eV} ; 0.12 \mathrm{eV}<$ center-of-mass energy $<12.10 \mathrm{eV}$ ), followed by a $3-\mathrm{s}$ delay to allow the pressure in the analyzer trap to drop to $0.7 \times 10^{-8}$ torr. CID results in loss of $C_{2}$ and $C_{4}$ from the parent to generate $\mathrm{C}_{92}^{+}$and $\mathrm{C}_{90}^{+}$as shown in Figure $5 \mathrm{c}(500-\mu$ s resonant excitation time, laboratory frame energy $88 \mathrm{eV}$, center-of-mass energy $3.0 \mathrm{eV}$ ). Under these conditions, $\sim 14 \%$ of the $\mathrm{C}_{94}^{+}$parent ions are converted into fragments; this value rises to $\sim 27 \%$ at $6-\mathrm{eV}$ center-of-mass energy. Loss of only even numbers of carbon atoms is consistent with the fullerene structure, as observed previously both by photodissociation [52] and CID [53-61]. Conversion of the parent ion $\mathrm{C}_{94}^{+}$into $\mathrm{C}_{92}^{+}$and $\mathrm{C}_{90}^{+}$is the only process observed,

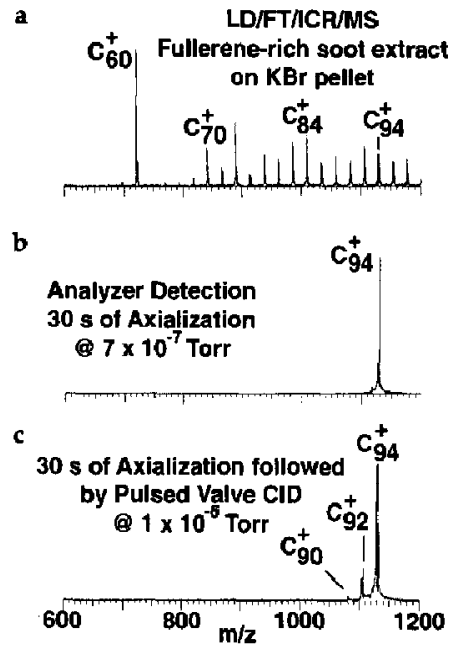

Figure 5. Nd:YAG laser desorption-ionization FTICR mass spectra of $\mathrm{C}_{94}^{+}$ions from fullerene soot sublimed on a $\mathrm{KBr}$ pellet. (a) Broadband detection in the source trap at $6 \times 10^{-8}$ torr. (b) Axialization of $\mathrm{C}_{9_{4}}^{+}$ions in the source trap by quadrupolar excitation $(11 \mathrm{~V}(\mathrm{p}-\mathrm{p})$ at $40.4 \mathrm{kHz})$ at $7 \times 10^{-7}$ torr for $30 \mathrm{~s}$, followed by transfer to the analyzer trap for detection at $0.4 \times$ $10^{-8}$ torr. (c) Axialization and transfer of $C_{94}^{1}$ ions for $30 \mathrm{~s}$ in the source trap under the same conditions as in (b), followed by pulsed valve admission of argon gas for CID in the analyzer trap at $\sim 1 \times 10^{-6}$-torr pressure (88-eV laboratory frame energy, 3.0$\mathrm{eV}$ center-of-mass energy), followed $3 \mathrm{~s}$ later by dipolar excitation-dipolar detection in the analyzer trap at $0.7 \times 10^{-8}$ torr. up to the onset of radial ejection; smaller fullerenes are not observed, presumably because the $\mathrm{C}_{94}^{+}$parent ion does not attain sufficient translational energy for further CID.

Finally, we note that subsequent to the presently demonstrated enhancement of tandem mass spectrometry by selective axialization of parent ions [62], Guan and coworkers [11] showed that CID product ion detection may be enhanced by axialization of the product ions. Thus, based on these two complementary approaches, it appears that axialization can enhance significantly the performance (parent isolation mass selectivity, CID efficiency, and product ion detection efficiency) in both stages of tandem mass spectrometry. In fact, an $\mathrm{MS}^{4}$ series of CID experiments starting from bradykinin quasimolecular $(\mathrm{M}+\mathrm{H})^{+}$ions has recently been reported [63].

\section{Acknowledgments}

We thank R. Ziolo of Xerox Corporation for his gift of $\mathrm{KH}$ soot, and J. V. Coe of The Ohio State University for providing toluene-extracted arc-welded soot. This work was supported by the National Science Foundation (CHE-90-21058), the Public Health Service (NIH GM-31683), The Ohio State University, and the National High Magnetic Field Laboratory at Florida State University.

\section{References}

1. Williams, E. R.; Henry, K. D; McLafferty, F. W. I. Am. Chem. Soc. 1990, 112, 6157-6162.

2. Maruyama, S.; Anderson, L. R.; Smalley, R. E. Rev. Sci. Instrum. 1990, 61, 3686-3693.

3. Brown, L. S.; Gabrielse, G. Rev. Mod. Phys, 1986, 58, 233-311.

4. Littlejohn, D. P.; Ghaderi, S. U.S. Patent No. 4,581,533, issued April 8, 1986.

5. Marshall, A. G.; Comisarow, M. B.; Parisod, G. J. Chem. Phys. $1979,77,4434-4444$.

6. Francl, T. J.; Fukuda, E. K.; McIver, R. T., Jr. Int. J. Mass Spectrom. Ion Processes 1983, 50, 151-167.

7. Bollen, G.; Moore, R. B.; Savard, G.; Stolzenberg, H. Appl. Phys. 1990, 68, 4355-4374.

8. Savard, G.; Becker, S.; Bollen, G.; Kluge, H.-J.; Moore, R. B.; Schweikhard, L.; Stolzenberg, H.; Wiess, U. Phys. Lett. A 1991, 158. 247-252.

9. Schweikhard, L.; Guan, S.; Marshall, A. G. Int. J. Mass Spectrom. Ion Processes 1992, 120, 71-83.

10. Guan, S.; Wahl, M. C.; Wood, T. D.; Marshall, A. G. Anal. Chem. 1993, 65, 1753-1757.

11. Guan, S.; Marshall, A. G.; Wahl, M. Anal. Chem. 1994, 66, 1363-1367.

12. Speir, J. P.: Gorman, G. S.; Pitsenberger, C. C.; Turner, C. A.; Wang, P. P.; Amster, I. J. Antal. Chem. 1993, 65, 1746-1752.

13. Guan, Z; Hoftstadler, 5. A.; Laude, D. A., Jr. Anal. Chem. $1993,65,1588-1593$.

14. Guan, S.: Marshall, A. G. J. Chem. Phys. 1993, 98, 4486-4493.

15. Guan, S.; Wah1, M. C.; Marshall, A. G. J. Chem. Phys. 1994, $100,6137-6140$.

16. Guan, S.; Wahl, M. C.; Marshall, A. G. Anal. Chem. 1993, 65, $3647-3653$.

17. Guan, S.; Marshall, A. G. Rapid Commun. Mass Spectrom. 1993, $7,857 \div 860$ 
18. Marshall, A. G.; Wang, T.-C. L.; Ricca, T. L. J. Am. Chem. Soc. 1985, 107, 7893-7897.

19. Wang, T.-C. L.; Ricca, T. L.; Marshall, A. G. Anal. Chem. 1986, $58,2935-2938$

20. Marshall, A. G.; Wang, T.-C. L; Chen, L.; Ricca, T. L. In M. V. Buchanan, Ed.; Fourier Transform Mass Spectrometry: Evolution, Innovation, and Applications; ACS Symposium Series 359; American Chemical Society: Washington, DC, 1987; pp 21-33.

21. Hsu, A. T.; Hunter, W. W., Jr.; Schmalbrock, P.; Marshall, A. G. J. Magrn. Reson. 1987, 72, 75-88.

22. McLafferty, F. W., Ed. Tandem Mass Spectrometry; Wiley: New York, 1983.

23. Krätschmer, W.; Lamb, L. D.; Fostiropooulos, K.; Huffman, D. R. Nature (London) 1990, 347, 354-358.

24. Lamb, L. D.; Huffman, D. R.; Workman, R. K.; Howells, S.; Chen, T.; Sarid, D.; Ziolo, R. F. Science 1992, 255, 1413-1416.

25. Haufler, R. E.; Conceicao, J.; Chibante, L. P. F.; Chai, Y; Byrne, N. E.; Flanagan, S.; Haley, M. M.; O'Brien, S. C.; Pan, C.; Xiao, Z.; Billups, W. E.; Ciufolini, M. A.; Hauge, R. H.; Margrave, J. L.; Wilson, L. J.; Curl, R. F.; Smalley, R. E. $J$. Phys. Chem. 1990, 94, 8634 .

26. Liang, Z.; Ricca, T. L.; Marshall, A. G. Rapid Commun. Mass Spectrom. 1991, 5, 132-136.

27. Cody, R. B.; Burnier, R. C.; Freiser, B. S. Anal. Chem. 1982, 54, 96-101.

28. Wise, M. B. Anal. Chem. 1987, 59, 2289-2293.

29. Cody, R. B. Analusis 1988, 16, 30-36.

30. Freiser, B. S. Talanta 1985, 32, 697-708.

31. Guan, S.; Xiang, X.; Marshall, A. G. Int. J. Mass Spectrom. Ion Processes 1993, 124, 53-67.

32. Schweikhard, L.; Marshall, A. G. J. Am. Soc. Mass Spectrom. $1993,4,433-452$

33. Chen, R.; Guan, S.; Marshall, A. G. I. Chem. Phys. 1994, 100, 2258-2266.

34. Marshall, A. G.; Verdun, F. R. Fourier Transforms in NMR, Optical, and Mass Spectrometry: A User's Handbook; Elsevier: Amsterdam, 1990.

35. Marshall, A. G.; Roe, D. C. J. Chem. Phys. 1980, 73, 1581-1590.

36. Guan, S. I. Am. Soc. Mass Spectrom. 1991, 2, 483-486.

37. Grosshans, P. B.; Marshall, A. G. Anal. Chem. 1991, 63, 2057-2061.

38. Jeffries, J. B.; Barlow, S. E.; Dunn, G. H. Int. J. Mass Spectrom. Ion Processes 1983, 54, 169-187.

39. McLafferty, F. W.; Amster, I. J.; Furlong, J. J. P.; Loo, J. A.; Wang, B. H.; Williams, E. R. In M. V. Buchanan, Ed.; Fourier Transform Mass Spectrometry: Evolution, Innovation, and Applications; ACS Symposium Series 359; American Chemical Society: Washington, DC, 1987; pp 116-126.
40. Gross, M. L.; Rempel, D. L. Science 1984, 226, 261-268.

41. Nibbering, N. M. M. Analyst 1992, 117, 289-293.

42. Comisarow, M. B.; Grassi, V.; Parisod, G. Chem. Phys. Letr. $1978,57,413-416$.

43. Mullen, S. L.; Marshall, A. G. J. Am. Chem. Soc. 1988, 110, 1766-1774.

44. Ross, C. W., III; Guan, S; Grosshans, P. B.; Ricca, T. L.; Marshall, A. G. J. Am. Chem. So. 1993, 115, 7854-7861.

45. Williams, E. R.; Loh, S. Y.; McLafferty, F. W.; Cody, R. B. Anal. Chem. 1990, 62, 698-703.

46. Grosshans, P. B.; Shields, P. J.; Marshall, A. G. J. Am. Chem. Soc. 1990, 112, 1275-1277.

47. Grosshans, P. B.; Marshall, A. G. Int. I. Mass Spectrom. Ion Processes 1990, 100, 347-379.

48. Grosshans, P. B.; Marshall, A. G. Int. I. Mass Spectrom. Ion Processes 1992, 115, 1-19.

49. Gauthier, J. W.; Trautman, T. R.; Jacobson, D. B. Anal. Chim. Acta 1991, 246, 211-225.

50. Diederich, F.; Whetten, R. L. Acc. Chem. Res. 1992, 25, 119-126.

51. Zhang, B. L; Wang, C. Z; Ho, K. M. Chem. Phys. Lett. 1992, 193, 225-230.

52. O'Brien, S. C.; Heath, J. R.; Curl, R. F.; Smalley, R. E. J. Chem. Phys. 1988, 88, 220-230.

53. Luffer, D. R, Schram, K. H. Rapid Commun. Mass Spectrom. $1990,4,552-556$.

54. Young, A. B.; Cousins, L. M.; Harrison, A. G. Rapid Commun. Mass Spectrom. 1991, 5, 226-229.

55. Ben-Amotz, D.; Cooks, R. G.; Dejarme, L.; Gunderson, J. C.; Hoke, S. H., II; Kahr, B.; Payne, G. L.; Wood, J. M. Chem. Phys. Lett, 1991, 183, 149-152.

56. Weiske, T.; Böhme, D. K.; Hrusák, J.; Krätschmer, W.; Schwarz, H. Angew. Chem. Int. Ed. Engl. 1991, 30, 884.

57. Weiske, T,; Böhme, D. K.; Schwarz, H. J. Phys. Chem. 1991, 95, 8451-8452

58. Lifshitz, C.; Iraqii, M,; Peres, T.; Fischer, J. E. Int. J. Mass Spectrom. Ion Processes 1991, 107, 565-569.

59. Caldwell, K. A.; Giblin, D. E; Hsu, C. S.; Cox, D.; Gross, M. L. J. Am. Chem. Soc. 1991, 113, 8519-8521.

60. Caldwell, K. A.; Giblin, D. E.; Gross, M. L. J. Am. Chem. Soc 1992, 114, 3743-3756

61. Ross, M. M.; Callahan, J. H. J. Phys. Chem. 1991, 95, 5720-5723.

62. Wood, T. D. Ph.D. Thesis, The Ohio State University, 1993.

63. Paša-Tolíc, L.; Huang, Y.; Kim, H. S; Guan, S.; Marshall, A. G. 42nd Amer. Soc. Mass Spectrom. Conf. on Mass Spectrometry \& Allied Topics; Chicago, IL, May 1994, WP 96. 